\title{
Amine Analysis Using AlexaFluor 488 Succinimidyl Ester and Capillary Electrophoresis with Laser-Induced Fluorescence
}

\author{
Christian G. Kendall, ${ }^{1,2}$ Amanda M. Stockton, ${ }^{1,3}$ Stephen Leicht, ${ }^{1,4}$ Heather McCaig, \\ Shirley Chung, ${ }^{1}$ Valerie Scott, ${ }^{1}$ Fang Zhong, ${ }^{1}$ and Ying Lin $^{1}$ \\ ${ }^{1}$ Jet Propulsion Laboratory, California Institute of Technology, Pasadena, CA 91109, USA \\ ${ }^{2}$ Weill Cornell Graduate School of Medical Science, New York, NY 10065, USA \\ ${ }^{3}$ Georgia Institute of Technology, Atlanta, GA 30332, USA \\ ${ }^{4}$ University of California, Los Angeles, CA 90095, USA
}

Correspondence should be addressed to Fang Zhong; fang.zhong@jpl.nasa.gov and Ying Lin; ying.lin@jpl.nasa.gov

Received 2 December 2014; Revised 23 February 2015; Accepted 24 February 2015

Academic Editor: Chih-Ching Huang

Copyright (C) 2015 Christian G. Kendall et al. This is an open access article distributed under the Creative Commons Attribution License, which permits unrestricted use, distribution, and reproduction in any medium, provided the original work is properly cited.

\begin{abstract}
Fluorescent probes enable detection of otherwise nonfluorescent species via highly sensitive laser-induced fluorescence. Organic amines are predominantly nonfluorescent and are of analytical interest in agricultural and food science, biomedical applications, and biowarfare detection. Alexa Fluor $488 \mathrm{~N}$-hydroxysuccinimidyl ester (AF488 NHS-ester) is an amine-specific fluorescent probe. Here, we demonstrate low limit of detection of long-chain $\left(\mathrm{C}_{9}\right.$ to $\left.\mathrm{C}_{18}\right)$ primary amines and optimize AF488 derivatization of long-chain primary amines. The reaction was found to be equally efficient in all solvents studied (dimethylsulfoxide, ethanol, and $\mathrm{N}, \mathrm{N}$-dimethylformamide). While an organic base (N,N-diisopropylethylamine) is required to achieve efficient reaction between AF488 NHS-ester and organic amines with longer hydrophobic chains, high concentrations $(>5 \mathrm{mM})$ result in increased levels of ethylamine and propylamine in the blank. Optimal incubation times were found to be $>12 \mathrm{hrs}$ at room temperature. We present an initial capillary electrophoresis separation for analysis using a simple micellar electrokinetic chromatography (MEKC) buffer consisting of $12 \mathrm{mM}$ sodium dodecylsulfate (SDS) and $5 \mathrm{mM}$ carbonate, $\mathrm{pH}$ 10. Limits of detection using the optimized labeling conditions and these separation conditions were 5-17 $\mathrm{nM}$. The method presented here represents a novel addition to the arsenal of fluorescent probes available for highly sensitive analysis of small organic molecules.
\end{abstract}

\section{Introduction}

Quantitative compositional analysis of specific primary amines is applied in food science and agriculture to characterize samples for quality control. Primary amines in soil samples provide information about the available sources of organic and bioorganic $\mathrm{N}$ in an ecosystem [1], a boon for management of agriculture, and a central aspect to researching systems that involve $\mathrm{N}$ or the nitrogen fixation cycle. Primary amines and amino acids are also used to indicate reactions in food processing and to directly indicate nutritional value and quality of products [2-5]. A large majority of known bioactive molecules and neurotransmitters are primary amines, amino acids, or low molecular weight metabolites of these species [6], so primary amine analysis is of continually increasing interest for metabolomics, pharmaceuticals, and detection of hazardous agents in biowarfare. In situ analysis of primary amines is additionally of great interest for investigating planetary chemistry $[7,8]$ as well as the synthesis and origin of prebiotic amino acids [9].

For compositional amine analysis, a separation method must typically be applied to resolve specific amines within a sample. Many applications require field-deployable amine compositional analyses, such as clinical devices, detection of harmful biological agents, and in situ astrobiology and planetary science experiments. Capillary electrophoresis (CE), when used in conjunction with laser-induced fluorescence (LIF), provides a fast and easily miniaturizable technique. CELIF also provides the opportunity to incorporate the separation and detection steps in line with extraction instruments 
and microfluidics devices. This is an attractive feature for lab-on-a-chip approaches and a necessary step for developing field-deployable detection instruments for the clinic, biowarfare, and in situ astrobiology experiments. In fact, CE is already a targeted implementation of lab-on-a-chip analysis for bioterrorism defense [10], $\mu$ CE-LIF has been fully automated and miniaturized towards future in situ Martian and other planetary missions $[7,11]$, and CE-LIF's direct compatibility with dialysates and biological fluids has been applied to clinical samples and in vivo biomedical research $[2,12,13]$. However, the latter two applications often require detection of amines with varied solubility in aqueous media. Micellar electrokinetic chromatography (MEKC) enables separation and therefore analysis of hydrophobic longer-chain amines, while preserving analytic capability of shorter and more hydrophilic amines. MEKC-LIF, only differing from CELIF by addition of a surfactant, presents the opportunity to extend implementation of these established approaches to field-deployable instrument development to detect a broader spectrum of targets in complex samples.

Fluorescence detection of primary amines provides a quick and potentially highly sensitive, quantitative analysis. Particularly, fluorescence detection of amines by CE-LIF with excitation at $488 \mathrm{~nm}$ has demonstrated limits of detection (LOD) from $\mu \mathrm{M}$ to $\mathrm{nM}[3]$ depending on the target amine, fluorescence probe, and optimization conditions. The speed of fluorescence detection ensures that the rate-limiting step for analysis is upstream in sample collection, preparation, or separation. However, to use this method, amines without autofluorescent properties must be chemically derivatized with a fluorescent probe. The commercially available dye, AlexaFluor 488 (AF488), is optimally excited with a $488 \mathrm{~nm}$ laser line (extinction coefficient of $73,000 \mathrm{~cm}^{-1} \mathrm{M}^{-1}$ at $494 \mathrm{~nm}$ ) and has an emission maximum at $525 \mathrm{~nm}$, making it easy to apply with standard light sources, instrumental settings, and filters. Low AF488 self-quenching enables highly sensitive analysis, and the N-hydroxysuccinimidyl ester (NHS-ester) functionality makes it highly aminespecific. In aqueous and biological samples, the spontaneous derivatization reaction of AF488 NHS-ester circumvents the need for additional reagents while the high amine specificity provides advantages over other amine-reactive dyes such as isothiocyanates, which also react with sulfhydryl groups, and dyes which react directly with amines and change their chemical structure and fluorescent properties upon derivatization (e.g., 4-chloro-7-nitro-1,2,3-benzoxadiazole). The derivatization through amine esterification, promoted by the NHS leaving group, allows for modular selection of other fluorophores to optimize fluorescent properties for varied equipment. AF488 is a water soluble probe that is $\mathrm{pH}$ insensitive over a range that extends from below 4 to above 10 , making it suitable for a range of applications. The dye is negatively charged overall, facilitating separation of tagged analytes by electromigration.

Here, we describe a novel method for amine analysis using MEKC-LIF in conjunction with a labeling protocol employing AF488 NHS-ester. We prepared and analyzed samples of nonylamine, hexadecylamine, and octadecyl amine to test the applicability of this method to aliphatic amines with reduced solubility in aqueous media and no detectable autofluorescence. Samples were separated and detected using a commercial Beckman Coulter P/ACE MDQ system with $488 \mathrm{~nm}$ LIF detection. Labeling conditions were optimized, including organic solvent, the concentration of the base diisopropylethylamine (DIEA), and the incubation time. Suitable separation characteristics were found using MEKC with sodium dodecyl sulfate as the surfactant, and the resulting analytical technique was characterized.

\section{Materials and Methods}

2.1. Materials. All chemicals were of analytical reagent grade and were used as received. AlexaFluor 488 succinimidyl ester (AF488 NHS-ester) was purchased from Invitrogen Corporation (Carlsbad, CA), diluted to $20 \mathrm{mM}$ in N,Ndimethylformamide (DMF, Sigma-Aldrich, St. Louis, MO), and stored at $-20^{\circ} \mathrm{C}$. Sodium carbonate $\left(\mathrm{NaCO}_{3}\right.$, SigmaAldrich) was used to prepare $50 \mathrm{mM}$ aqueous solutions with $18 \mathrm{M} \Omega \cdot \mathrm{cm}$ water. The $\mathrm{pH}$ was adjusted using $1 \mathrm{M}$ $\mathrm{NaOH}$ (Sigma-Aldrich) and measured using a glass electrode and a digital $\mathrm{pH}$ meter (Orion 290A, Thermo; Waltham, MA). Sodium dodecyl sulfate (SDS) was acquired from Sigma Aldrich and used to prepare a $100 \mathrm{mM}$ stock in $18 \mathrm{M} \Omega \cdot \mathrm{cm}$ water. Amines for standard solutions, including nonylamine $\left(\mathrm{C} 9-\mathrm{NH}_{2}\right)$, dodecylamine $\left(\mathrm{C} 12-\mathrm{NH}_{2}\right)$, hexadecylamine $\left(\mathrm{C} 16-\mathrm{NH}_{2}\right)$, and octadecylamine $\left(\mathrm{C} 18-\mathrm{NH}_{2}\right)$, were purchased in pure form from Sigma Aldrich and used to prepare $10 \mathrm{mM}$ solutions in ethanol (Sigma-Aldrich). N,Ndiisopropylethylamine (DIEA, Sigma-Aldrich) was diluted to $10 \mathrm{mM}$ in ethanol, DMF, and dimethylsulfoxide (DMSO, Sigma-Aldrich). The stock solutions were combined as needed to result in the solutions used.

2.2. Labeling Reactions. Labeling reactions were conducted by combining the appropriate volumes of $20 \mathrm{mM}$ AF488, $10 \mathrm{mM}$ DIEA, amine, and solvent. Reactions were incubated in the dark overnight (16-24 hrs) unless otherwise indicated. After incubation, reactions were diluted into the separation buffer at a 5:100 ratio unless otherwise indicated.

2.3. Capillary Electrophoresis. Capillary electrophoresis (CE) separations were conducted on a Beckman Coulter P/ACE MDQ capillary electrophoresis system equipped with $488 \mathrm{~nm}$ laser-induced fluorescence (LIF) detection. The capillary was rinsed using pressure with the separation buffer for two (2) minutes, and then the sample was injected (pressure) for 5 seconds. Separations were conducted at $15 \mathrm{kV}$ for 15 minutes. After separation, the capillary was rinsed using pressure with pure water for five minutes. Capillary conditioning using $1 \mathrm{M} \mathrm{NaOH}$ was conducted with a 5-minute rinse as needed. Separation buffers tested included $10 \mathrm{mM}$ carbonate ( $\mathrm{pH} 10)$ and $10 \mathrm{mM}$ carbonate with $12 \mathrm{mM}$ SDS ( $\mathrm{pH} 10)$.

2.4. Data Analysis and Figure Generation. Resulting electropherograms were generated and exported in commaseparated values format using 32 Karat software (Beckman Coulter Inc.). These files were imported into PeakFit (Systat) 
<smiles>CCC(C)C(=O)ON1C(=O)CCC1=O</smiles>

(a)
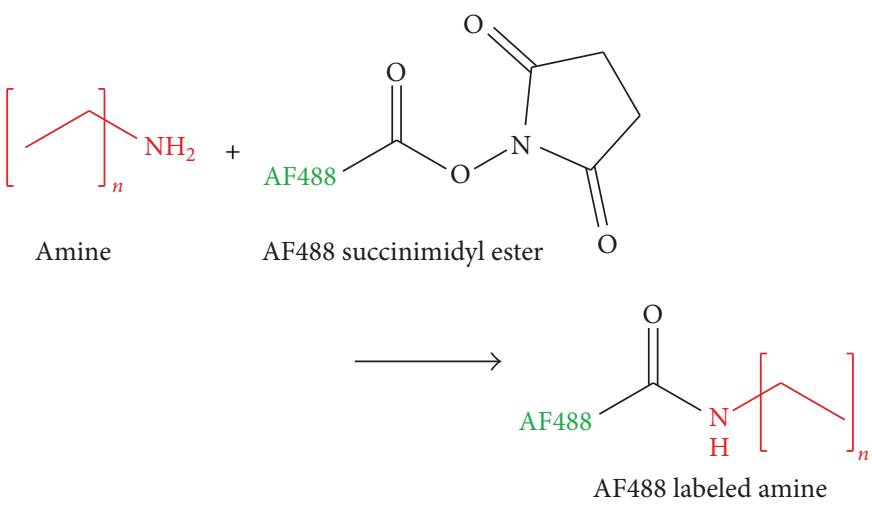

(b)

Figure 1: AlexaFluor 488 (AF488) succinimidyl ester and its reaction with primary amines. (a) The chemical structure of the reaction of AF488 succinimidyl ester with primary amines. N-hydroxysuccinimide acts as a leaving group to promote the formation of an amide bond to link AF488 to the primary amine group.

for smoothing ( $0.1 \%$ Loess) and baseline correction prior to peak fitting. The resulting smoothed and baseline corrected electropherograms or data from peak fitting was imported into Origin (OriginLabs) to generate figures. Chemical equations were drawn in ChemBioDraw Ultra. All raw figures were imported into Adobe Illustrator for image cleanup.

\section{Results and Discussion}

Figure 1 shows the labeling reaction between AF488 NHSester and a primary amine. This reaction is base-catalyzed and was found to proceed for $\mathrm{C} 9-\mathrm{NH}_{2}$ and shorter amines in $10 \mathrm{mM}$ aqueous carbonate, $\mathrm{pH} 10$. Longer-chain amines $\left(\mathrm{C} 12-\mathrm{NH}_{2}\right.$ and longer) were found to be insoluble in aqueous solutions without surfactant and therefore did not label to any detectable extent. For this reason, we examined organic solvents for labeling reactions with DIEA included to provide a basic environment. The fluorescence intensities of amines labeled in $10 \mathrm{mM}$ DIEA in ethanol, DMF, and DMSO and then separated in $10 \mathrm{mM}$ carbonate, $12 \mathrm{mM}$ SDS, $\mathrm{pH}$ 10, are shown to be normalized to the DMSO fluorescence intensity in Figure 2. Labeling proceeded to nearly the same extent, within error, in all three organic solvents. DMSO may provide slightly better labeling than ethanol. DMSO is often favored in extraction and sample preparation for its solvating ability and stability, so it is encouraging to see that labeling proceeded optimally in DMSO. However, these results also indicate that choice of solvent can be dictated by concerns such as downstream analysis method, safety, and ease of evaporation with marginal reduction in labeling.

To explore the impact of DIEA concentration on labeling efficiency, its concentration in ethanol was varied from 0 to $48.75 \mu \mathrm{M}$ in a solution that contained $1 \mu \mathrm{M}$ amine and $25 \mu \mathrm{M}$ AF488 NHS-ester. Figure 3 shows the results of CE separation after an overnight incubation of the solutions. While very low levels of amine were labeled without any DIEA, there is no change, within error, of the amount labeled in solutions containing between 12.5 and $48.75 \mu \mathrm{M}$ DIEA. However, some contamination in the shorter chain amine region $\left(\mathrm{C} 2-\mathrm{NH}_{2}, \mathrm{C} 3-\mathrm{NH}_{2}\right)$ was observed to increase with increasing DIEA concentration. These results indicate that DIEA concentration can and, when possible, should be kept to a minimum.

While overnight incubations are logistically simple for an operator and for potential automated implementations, 
TABLE 1: Table of separation characteristics.

\begin{tabular}{|c|c|c|c|c|c|c|}
\hline Amine & Amplitude & Area & $\begin{array}{c}\text { Elution } \\
T^{\mathrm{a}}(\mathrm{min})\end{array}$ & $\begin{array}{l}\text { Peak efficiency } \\
\text { (Theor. plates) }\end{array}$ & $\begin{array}{l}\text { Peak effici. } \\
\text { (plates/m) }\end{array}$ & LOD (nM) \\
\hline Nonylamine & $2.2 \times 10^{7}$ & $3.5 \times 10^{7}$ & 5.5 & $2.7 \times 10^{5}$ & 550000 & N.D. ${ }^{b}$ \\
\hline Hexadecylamine & $4.5 \times 10^{7}$ & $1.1 \times 10^{8}$ & 7.0 & $1.7 \times 10^{5}$ & 350000 & 17 \\
\hline Octadecylamine & $4.4 \times 10^{7}$ & $1.1 \times 10^{8}$ & 7.0 & $1.1 \times 10^{5}$ & 220000 & 5.7 \\
\hline
\end{tabular}

${ }^{\mathrm{a}}$ Elution time.

${ }^{\mathrm{b}}$ N.D.: not determined.

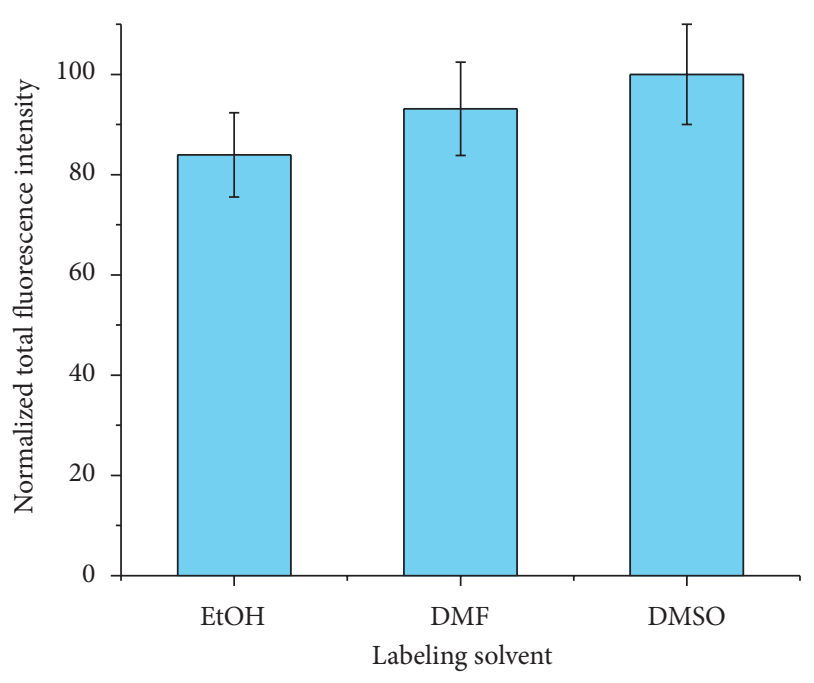

FIGURE 2: Normalized total fluorescence intensities of amines analyzed by CE-LIF and labeled with AF488 in ethanol (EtOH), dimethylformamide (DMF), and dimethylsulfoxide (DMSO). Labeling was conducted at $25 \mu \mathrm{M}$ amine, $50 \mu \mathrm{M}$ AF 488 with $1 \mathrm{mM}$ DIEA in the indicated solvent and incubated overnight. For analysis, the labeling solutions were diluted 1:20 in separation buffer $(10 \mathrm{mM}$ carbonate, $12 \mathrm{mM}$ SDS, $\mathrm{pH} 10$ ).

sometimes it is preferable to obtain results from a sample within a shorter window of overall time. Therefore, we examined the impact of incubation time on the labeling reaction extent. Figure 4 shows the relative fluorescence intensity of the amine peaks diluted into separation buffer and immediately separated via CE at incubation times from $1 \mathrm{hr}$ to $30 \mathrm{hr}$. Over $90 \%$ final intensity was achieved within 6 hours for the amines studied. While the samples were prepared in such a way to potentially yield pseudo-firstorder kinetics, the data do not fit a rate law first-order in amine concentration and in fact most closely fit a rate law second-order in amine concentration. This does not seem like a physical likelihood given stoichiometry of the reaction and the standard mechanism proceeding via a tetrahedral intermediate. Therefore, we cannot make any statements about true kinetic parameters of the reaction based on our data and recommend a true kinetics study as part of future work with the AF488 NHS-ester fluorescent probe.

Based on the above experiments, the optimum conditions for our work using AF488 NHS-ester as a fluorescent probe for amine analysis are $12.5 \mu \mathrm{M}$ DIEA in DMF with at least $6 \mathrm{hr}$ and preferably overnight incubation. Electropherograms

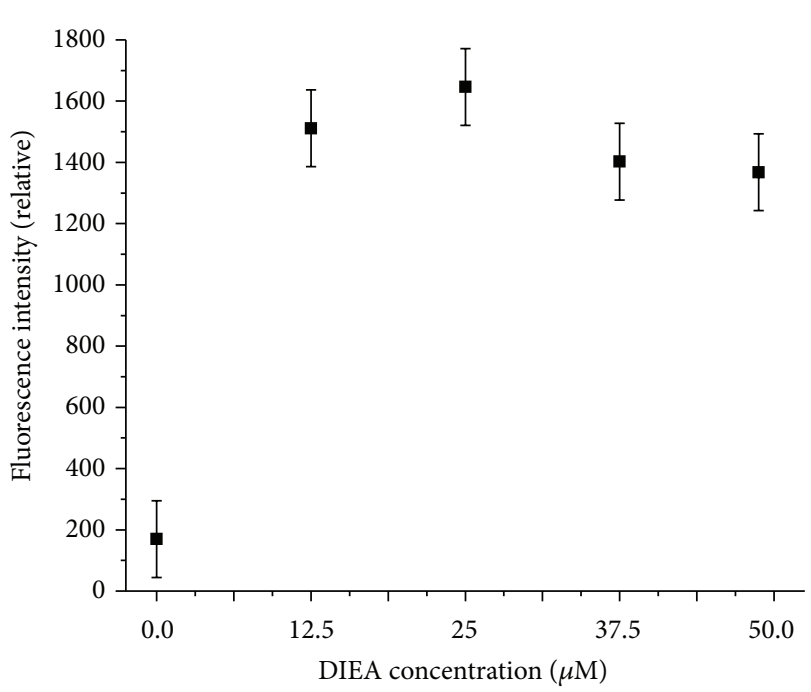

FIGURE 3: Effect of varying DIEA concentration on peak intensity (a measure of labeling efficiency). Relative fluorescence intensities of dodecylamine are plotted against varying DIEA concentration. Labeling was conducted at $1 \mu \mathrm{M}$ amine, $25 \mu \mathrm{M}$ AF488 with the indicated concentration of DIEA in ethanol and incubated overnight. For analysis, the labeling solutions were diluted $1: 10$ in separation buffer (10 mM carbonate, $12 \mathrm{mM}$ SDS, pH 10). Error bars are calculated from triplicate labeling at $12.5 \mu \mathrm{M}$.

of amines separated in $10 \mathrm{mM}$ carbonate, $12 \mathrm{mM}$ SDS, $\mathrm{pH}$ 10, after labeling with the optimized conditions, are shown in Figure 5. While the separation conditions were not optimized nor studied in great detail in this work, separation characteristics are given in Table 1 . Limits of detection $(S / N=$ 3) were determined using the optimized labeling conditions

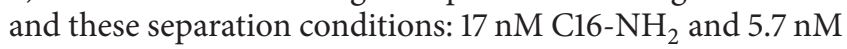
$\mathrm{C} 18-\mathrm{NH}_{2}$. A contamination issue with our $\mathrm{C} 9-\mathrm{NH}_{2}$ stock prevented determination of its LOD during the timeframe of this work. This work provides a foundation for further work more fully optimizing an AF488-based amine assay for a desired application.

\section{Conclusions}

Amine detection using LIF with AF488 as the reactive probe enables potentially novel analytical techniques as it provides an alternative to other probes' fluorescent at $488 \mathrm{~nm}$. The optimized reaction conditions are mild and fast $(90 \%$ efficiency within 6 hrs) while enabling excellent limits of 


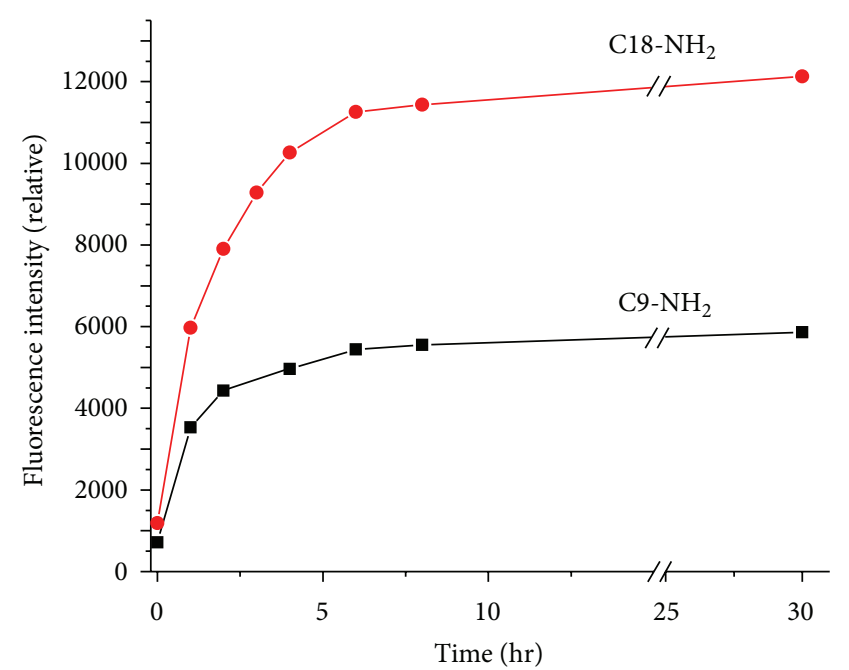

FIGURE 4: Effect of incubation time on labeling efficiency. Normalized fluorescence intensities of nonylamine and octadecylamine are plotted against incubation time. Labeling was conducted at $1 \mu \mathrm{M}$ amine, $25 \mu \mathrm{M}$ AF488 with $12.5 \mu \mathrm{M}$ DIEA in DMSO and incubated for the indicated time. For analysis, the labeling solutions were diluted $1: 20$ in separation buffer (10 mM carbonate, $12 \mathrm{mM}$ SDS, $\mathrm{pH}$ $10)$.
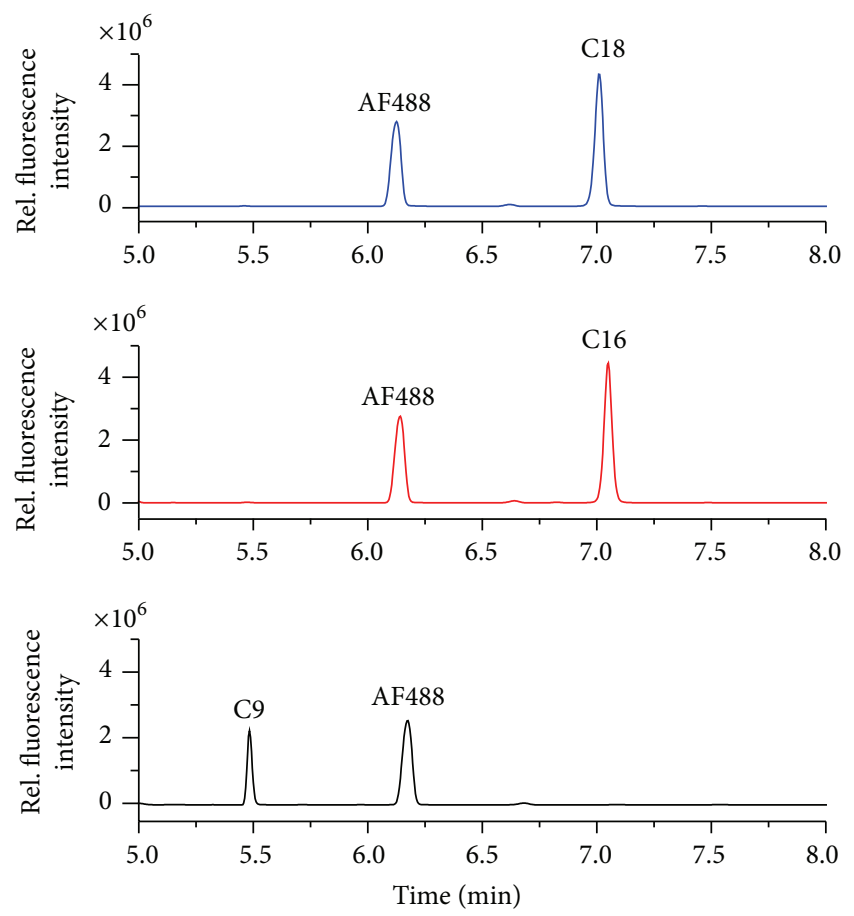

FIGURE 5: Electropherograms of amines labeled with the optimized labeling conditions ( $1 \mu \mathrm{M}$ amine, $25 \mu \mathrm{M}$ AF488 with $12.5 \mu \mathrm{M}$ DIEA in DMSO, and incubation for approximately 24 hours). For analysis, the labeling solutions were diluted 1:20 in separation buffer $(10 \mathrm{mM}$ carbonate, $12 \mathrm{mM}$ SDS, $\mathrm{pH} 10)$. detection (nM or ppb). While AF488 is not fluorogenic and therefore cannot be used as a fluorescent probe without some form of postreaction separation, we have demonstrated that this separation can be achieved using a simple, standard MEKC solution and a commercial CE instrument. The limits of detection achievable using the commercial system and initial CE separation conditions were in the low $\mathrm{nM}$ range, or single parts-per-billion, making this technique more than sufficiently sensitive for multiple applications. Further work remains to fully characterize the kinetics of the labeling reaction. Additionally, the current separation method is insufficient for the simultaneous analysis of C16$\mathrm{NH}_{2}$ and C18- $\mathrm{NH}_{2}$; thus, further work is required to develop application-specific optimized separation methods.

\section{Conflict of Interests}

The authors declare that there is no conflict of interests regarding the publication of this paper.

\section{Acknowledgments}

The research described in this paper was carried out at the Jet Propulsion Laboratory, California Institute of Technology, under a contract with the National Aeronautics and Space Administration (NASA) and was supported by the NASA Astrobiology Science and Technology Instrument Development program. The JPL author's copyright for this paper is held by the California Institute of Technology. Government sponsorship is acknowledged.

\section{References}

[1] C. R. Warren, "Organic N molecules in the soil solution: what is known, what is unknown and the path forwards," Plant and Soil, vol. 375, no. 1-2, pp. 1-19, 2014.

[2] H. F. Erbersdobler and V. Somoza, "Forty years of furosineforty years of using Maillard reaction products as indicators of the nutritional quality of foods," Molecular Nutrition and Food Research, vol. 51, no. 4, pp. 423-430, 2007.

[3] V. Poinsot, V. Ong-Meang, P. Gavard, and F. Couderc, "Recent advances in amino acid analysis by capillary electromigration methods, 2011-2013," Electrophoresis, vol. 35, no. 1, pp. 50-68, 2014.

[4] M. Lechtenberg, K. Henschel, U. Liefländer-Wulf, B. Quandt, and A. Hensel, "Fast determination of N-phenylpropenoyl-1amino acids (NPA) in cocoa samples from different origins by ultra-performance liquid chromatography and capillary electrophoresis," Food Chemistry, vol. 135, no. 3, pp. 1676-1684, 2012.

[5] D. Zhao, M. Lu, and Z. Cai, "Separation and determination of $B$ vitamins and essential amino acids in health drinks by CELIF with simultaneous derivatization," Electrophoresis, vol. 33, no. 15, pp. 2424-2432, 2012.

[6] G. Wu, "Amino acids: metabolism, functions, and nutrition," Amino Acids, vol. 37, no. 1, pp. 1-17, 2009.

[7] M. L. Cable, A. M. Stockton, M. F. Mora, and P. A. Willis, "Lowtemperature microchip nonaqueous capillary electrophoresis of aliphatic primary amines: applications to titan chemistry," Analytical Chemistry, vol. 85, no. 2, pp. 1124-1131, 2013. 
[8] M. L. Cable, S. M. Hörst, C. He et al., "Identification of primary amines in Titan tholins using microchip nonaqueous capillary electrophoresis," Earth and Planetary Science Letters, vol. 403, pp. 99-107, 2014.

[9] M. P. Callahan, A. S. Burton, J. E. Elsila et al., "A search for amino acids and nucleobases in the Martian meteorite Roberts Massif 04262 using liquid chromatography-mass spectrometry," Meteoritics and Planetary Science, vol. 48, no. 5, pp. 786-795, 2013.

[10] J. Wang, "Microchip devices for detecting terrorist weapons," Analytica Chimica Acta, vol. 507, no. 1, pp. 3-10, 2004.

[11] M. F. Mora, A. M. Stockton, and P. A. Willis, "Microchip capillary electrophoresis instrumentation for in situ analysis in the search for extraterrestrial life," Electrophoresis, vol. 33, no. 17, pp. 2624-2638, 2012.

[12] A. Zinellu, S. Sotgia, L. Deiana, and C. Carru, "Analysis of neurotransmitter amino acids by CE-LIF detection in biological fluids," Methods in Molecular Biology, vol. 919, pp. 35-42, 2013.

[13] C. J. Watson, R. Lydic, and H. A. Baghdoyan, "Sleep duration varies as a function of glutamate and GABA in rat pontine reticular formation," Journal of Neurochemistry, vol. 118, no. 4, pp. 571-580, 2011. 

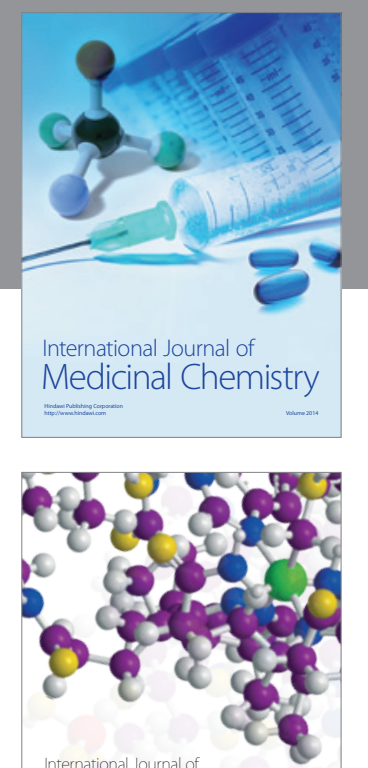

\section{Carbohydrate} Chemistry

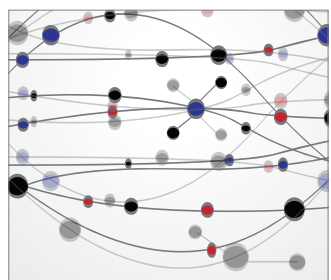

The Scientific World Journal
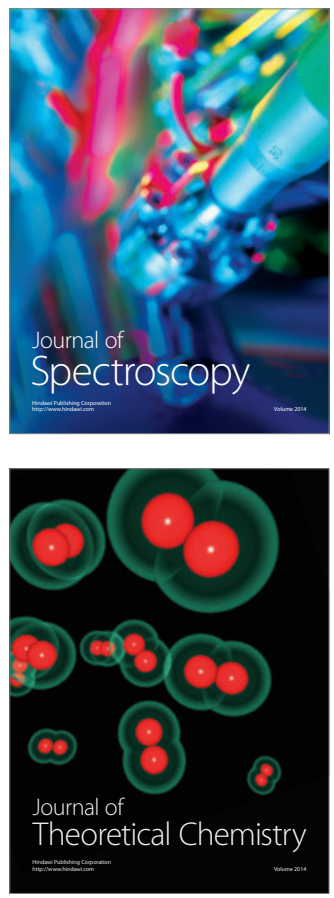
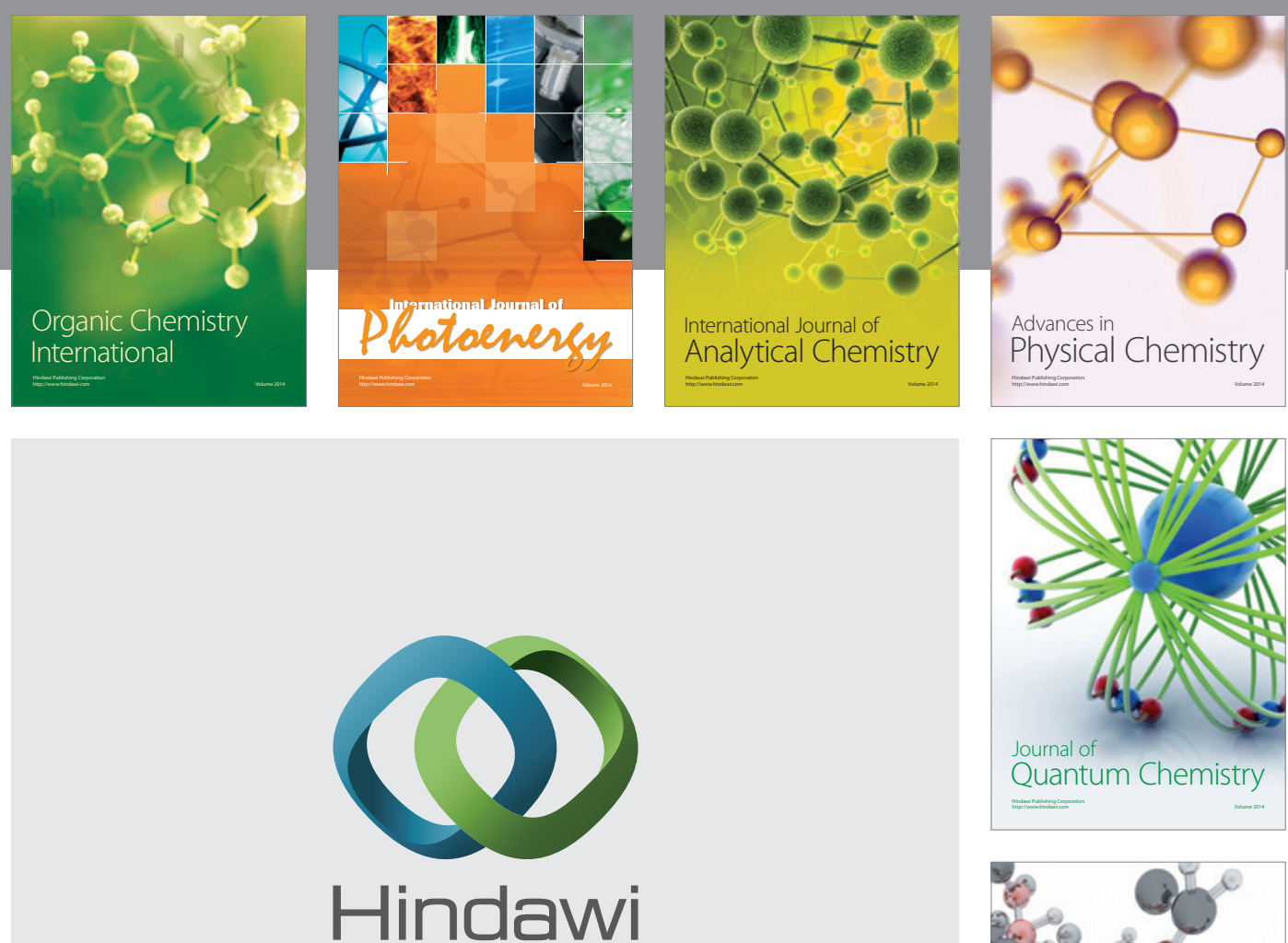

Submit your manuscripts at

http://www.hindawi.com

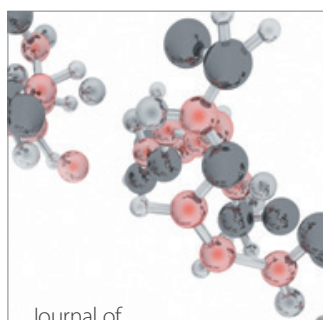

Analytical Methods

in Chemistry

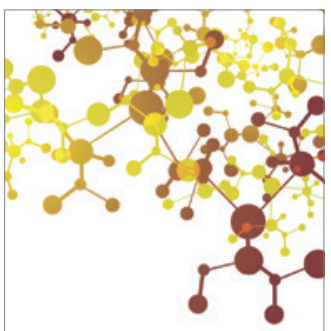

Journal of

Applied Chemistry

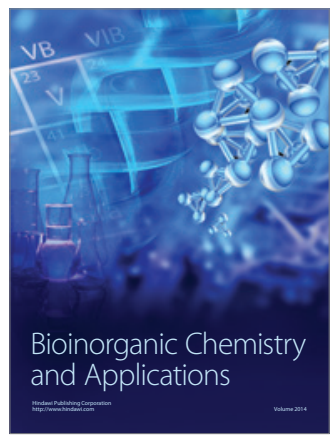

Inorganic Chemistry
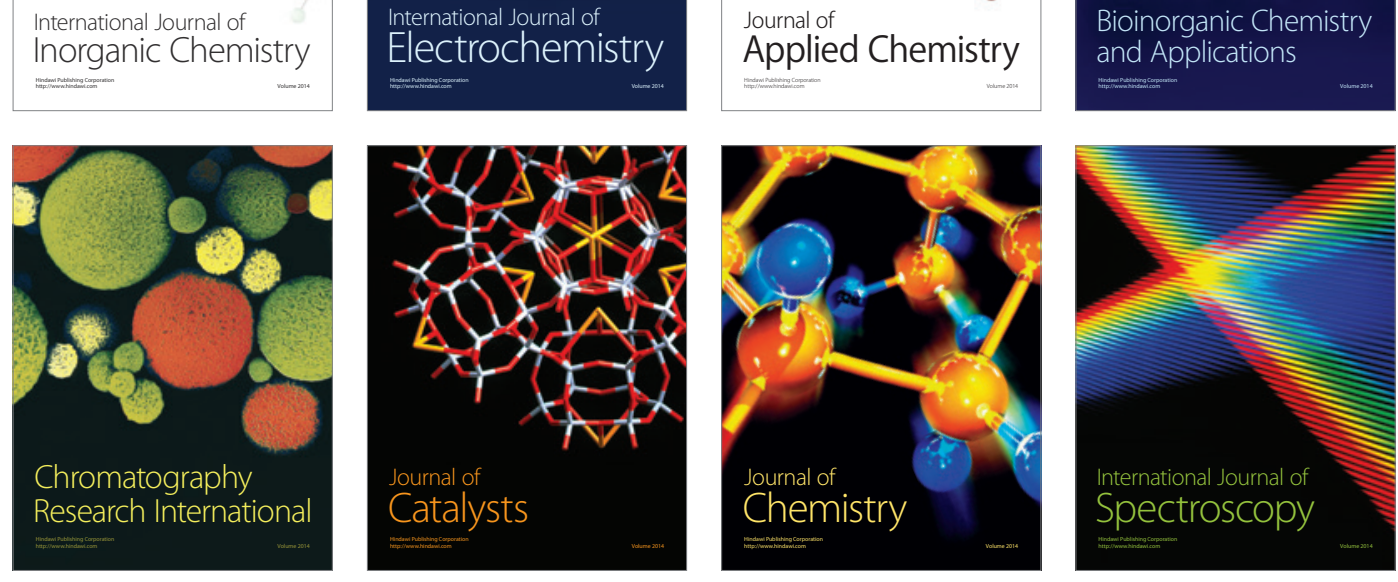